\title{
Association between sleeping difficulty and type 2 diabetes in women
}

\author{
Yanping $\mathrm{Li}^{1}$ • Xiang $\mathrm{Gao}^{2}$ • John W. Winkelman ${ }^{3,4}$ • Elizabeth M. Cespedes ${ }^{1,5,6}$ • \\ Chandra L. Jackson ${ }^{5,7}$ • Arthur S. Walters ${ }^{8}$ • Eva Schernhammer ${ }^{5}$. \\ Susan Redline ${ }^{5,9} \cdot$ Frank B. Hu ${ }^{1,5,10}$
}

Received: 23 September 2015 / Accepted: 17 December 2015 / Published online: 28 January 2016

(C) Springer-Verlag Berlin Heidelberg 2016

\begin{abstract}
Aims/hypothesis Sleeping difficulty has been associated with type 2 diabetes in some prior studies. Whether the observed associations are independent of health behaviours, other cardiovascular risk factors or other sleep disorders is unclear. Methods We analysed data from 133,353 women without diabetes, cardiovascular disease and cancer at baseline in the Nurses' Health Study (NHS, 2000-2010) and the NHSII (2001-2011). Sleeping difficulty was assessed as having difficulty falling or staying asleep 'all of the time' or 'most of the time' at baseline (2000 in NHS and 2001 in NHSII). Results We documented 6,407 incident cases of type 2 diabetes during up to 10 years of follow-up. After adjustment for lifestyle factors at baseline, comparing women with and without sleeping difficulty, the multivariate-adjusted HR (95\% CI) for type 2 diabetes was 1.45 (95\% CI 1.33, 1.58), which changed to $1.22(95 \%$ CI $1.12,1.34)$ after further adjustment for hypertension, depression and BMI based on the updated repeated measurements. Women who reported all four sleep conditions (sleeping difficulty, frequent snoring,
\end{abstract}

Frank B. Hu

nhbfh@channing.harvard.edu

1 Department of Nutrition, Harvard T. H. Chan School of Public Health, 665 Huntington Ave, Boston, MA 02115, USA

2 Department of Nutritional Sciences, The Pennsylvania State University, University Park, PA, USA

3 Department of Psychiatry, Harvard Medical School, Boston, MA, USA

4 Departments of Psychiatry and Neurology, Sleep Disorders Clinical Research Program, Massachusetts General Hospital, Boston, MA, USA sleep duration $\leq 6 \mathrm{~h}$ and sleep apnoea in NHS or rotating shift work in NHSII) had more than a fourfold increased likelihood of type 2 diabetes (HR 4.17, 95\% CI 2.93, 5.91).

Conclusions/interpretation Sleeping difficulty was significantly associated with type 2 diabetes. This association was partially explained by associations with hypertension, BMI and depression symptoms, and was particularly strong when combined with other sleep disorders. Our findings highlight the importance of sleep disturbance in the development and prevention of type 2 diabetes.

Keywords Diabetes · Pathways $\cdot$ Sleeping difficulty
Abbreviations
AHEI Alternate healthy eating index
NHS Nurses' Health Study
SES Socioeconomic status

5 Department of Epidemiology, Harvard T. H. Chan School of Public Health, Boston, MA, USA

6 Division of Research, Kaiser Permanente Northern California, Oakland, CA, USA

7 Harvard Catalyst $\mid$ Clinical and Translational Science Center, Boston, MA, USA

8 Vanderbilt University School of Medicine Medical Center, Nashville, TN, USA

9 Brigham and Women's Hospital and Beth Israel Deaconess Medical Center, Harvard Medical School, Boston, MA, USA

10 Channing Division of Network Medicine, Department of Medicine, Brigham and Women's Hospital and Harvard Medical School, Boston, MA, USA 


\section{Introduction}

Sleeping difficulty is a common disorder characterised by difficulty initiating or maintaining sleep [1,2], affecting approximately $10 \%$ to $20 \%$ of the general population in the USA [1]. In a study of US adults, the number of outpatient visits identified to be due to sleeping difficulty increased from 4.9 million in 1999 to 5.5 million in 2010 [3].

Experimental studies indicate that inadequate or fragmented sleep is associated with impaired glucose regulation in healthy young adults $[4,5]$. Sleep disturbance has been shown to adversely influence metabolism and has been increasingly linked to a wide range of adverse health outcomes, including obesity [6], hypertension [7] and depression [8], which are all risk factors for type 2 diabetes. A number of epidemiological studies have identified that difficulty initiating or maintaining sleep is associated with a significantly increased risk of type 2 diabetes [9-12]. However, it is not clear whether these associations are explained by the chronic conditions of obesity, hypertension or depression. Short sleep duration, snoring, sleep apnoea and shift work may co-occur with sleeping difficulty and associate with diabetes [13-18]. The extent to which prior associations between sleeping difficulty and diabetes reflects confounding with these other sleep conditions is unclear.

The high prevalence of both sleeping difficulty and type 2 diabetes points to a need to further understand the link between the two conditions $[1,19]$. In this study, we examined the association between sleeping difficulty, defined as having difficulty initiating or maintaining sleep most of the time or all the time [1,2], and the risk of developing type 2 diabetes based on the extracted data from two independent, wellestablished cohorts of US women: the Nurses' Health Study (NHS) [17] and NHSII [17, 18]. We also examined the joint effect between sleeping difficulty and short sleep duration, frequent snoring, sleep apnoea and rotating shift work. Additionally, we quantified the extent to which the effects of sleeping difficulty on type 2 diabetes are mediated through BMI, hypertension and depression.

\section{Methods}

Study population The NHS [17] was initiated in 1976 when 121,700 female registered nurses aged 30-55 years completed a mailed questionnaire. The NHSII cohort [18], established in 1989 , comprises 116,671 younger female registered nurses aged 25 to 42 years. The cohorts have been followed by means of biennial mailed questionnaires, which inquired about lifestyle practices and other exposures of interest, as well as the incidence of disease $[17,18]$.

The information about sleeping difficulty was derived from answers by participants who were actively participating in the
NHS surveys in the year $2000(n=82,468)$ or the NHSII surveys in the year $2001(n=86,006)$, which was the baseline of the proposed study. For the current analysis, we excluded participants who had ever been diagnosed with cancer $(n=20,283)$, cardiovascular disease $(n=4,876)$ or diabetes $(n=7,868)$ at baseline, or did not respond to the questions on sleeping difficulty $(n=1,021)$ at baseline or were lost to follow-up after baseline $(n=1,073)$, leaving 133,353 women in our primary analysis.

The Institutional Review Boards at the Harvard School of Public Health and Brigham and Women's Hospital approved the study protocol. The completion of the self-administered questionnaire was considered to imply informed consent.

Ascertainment of sleeping difficulty and other sleeprelated factors The nurses recorded 'how much of the time during the past 4 weeks have you had difficulty falling asleep or staying asleep?' in 2000 in NHS and 2001 in NHSII. The possible responses were as follows: 'all of the time', 'most of the time', 'a good bit of the time', 'some of the time', 'a little of the time' and 'none of the time'. In the current study, we classified the participants who reported difficulty falling asleep or staying asleep 'all of the time' or 'most of the time' as having sleeping difficulty whereas the others ('a good bit of the time', 'some of the time', 'a little of the time' and 'none of the time') were classified as not having sleeping difficulty.

Information on sleep duration and snoring frequency was collected in both NHS (2000) and NHSII (2001). Short sleep duration was classified as sleeping $\leq 6 \mathrm{~h} /$ day. Shift work, only recorded in NHSII at baseline, was assessed as whether the participant 'worked permanent night shifts for 6 or more months during 1995-2001'. Information about sleep apnoea was only recorded in 2008 in NHS. Physician diagnosis of sleep apnoea and year of the first diagnosis (1997 or before, 1998-2001, 2002-2005, 2006-2007 or 2008+) were only recorded in 2008 in NHS.

Ascertainment of type 2 diabetes Type 2 diabetes cases were defined as self-reported diabetes confirmed by a validated supplementary questionnaire [17, 18]. For cases before 1998, we used the National Diabetes Data Group criteria to define type 2 diabetes. We used the American Diabetes Association diagnostic criteria for type 2 diabetes diagnosis from 1998 onwards. The validity of self-reported type 2 diabetes diagnosis in our cohorts has been previously documented in detail $[20,21]$. Briefly, 97-98\% cases were reconfirmed against blinded physician medical record review [20,21].

Assessment of other covariates Information on potential confounders, such as age, ethnicity, smoking status, weight, height, physical activity, menopausal status and postmenopausal hormone therapy, multiple vitamin use, regular use of medications (e.g., aspirin, antidepressants, minor tranquilisers 
and antihypertensive drugs), and physician diagnosis of hypertension and depression was collected via biennial questionnaires. Information on food and alcohol consumption was collected every 4 years via a validated semi-quantitative food frequency questionnaire. BMI was calculated as weight $(\mathrm{kg}) /$ height ${ }^{2}(\mathrm{~m})$. Hypertension was considered as either professionally diagnosed hypertension or use of antihypertensive medications. A participant was considered as having depression if she reported physician-diagnosed depression or use of antidepressant medications. Dietary quality was assessed by the alternate healthy eating index (AHEI), based on a high consumption of vegetables, fruit, nuts and legumes, whole grains, long-chain fats, polyunsaturated fatty acids, and a low level of sugar-sweetened beverages and fruit juice, red/ processed meat, trans fats and sodium. At baseline, the nurses ranked their feeling about their standing in US society in 10 levels; those at the top are the people who are the best offthose who have the most money, the most education, and the most respectable jobs, while at the bottom are the people who are the worst off-those who have the least money, the least education, and the least respectable jobs or no job, which was used as a surrogate indicator of socioeconomic status (SES).

Statistical analysis Individuals contributed person-time from the return of the baseline questionnaire (NHS, 2000; NHSII, 2001) until the date of diagnosis of type 2 diabetes, death, loss to follow-up, or the end of the follow-up period (30 June 2010 for NHS and 30 June 2011 for NHSII), whichever came first.

Multivariable Cox proportional hazards models were used to estimate HR and $95 \%$ CIs of type 2 diabetes according to the 'frequency of having difficulty falling asleep or staying asleep'. A linear trend across the categories was quantified with a Wald test. We also presented the risk of type 2 diabetes comparing women with and without sleeping difficulty (dichotomy). Multivariable models were adjusted for the baseline levels of menopausal status and postmenopausal hormone therapy use, smoking status, alcohol consumption, physical activity, AHEI, ethnicity, family history of diabetes and self-reported SES status.

To examine the extent to which chronic conditions explained the association between sleeping difficulty and risk of type 2 diabetes, we estimated the magnitude of change in the regression coefficient for sleeping difficulty with and without adjustment for each individual and multiple chronic condition, including BMI, hypertension and depression history, which were updated every other year. The per cent of excessive risk explained by the chronic conditions was computed as follows: $\left(1-\left[\beta_{\text {chronic condition- }}\right.\right.$

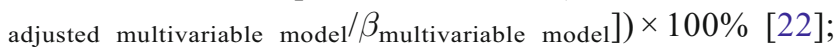
where $\beta_{\text {multivariable model }}$ referred to the basic multivariable beta-coefficient and $\beta_{\text {chronic condition-adjusted multivariable model }}$ referred to the beta-coefficient from the basic multivariable plus chronic conditions. A positive change in the regression coefficient indicates a change in the rate ratio towards the null. SAS macro \%MEDIATE was applied (publicly available at www.hsph.harvard.edu/faculty/ spiegelman/mediate.html).

Participants were also classified according to the joint categories of sleeping difficulty and other sleep-related conditions, including sleep duration, frequent snoring (every night or most nights), rotating shift work (worked permanent night shifts for 6 or more months in last 6 years), and sleep apnoea (physician diagnosed vs not). We tested the interaction using multiplicative and additive interaction analyses [23]. The multiplicative interaction was carried out by comparing the $-2 \log$ likelihood of the models with and without interaction term; while the additive interaction was testing by estimation of Relative Excess Risk due to Interaction [23].

In considering the effect of potential reverse causality between sleeping difficulty and chronic conditions, we did a sensitivity analysis among a relative healthy population who had a BMI less than $30 \mathrm{~kg} / \mathrm{m}^{2}$ without physician diagnosis of either hypertension or depression, and with neither antihypertensive nor antidepressant medications at baseline.

The main analysis was based on the pooled data from the two cohorts (NHS and NHSII) after further adjusting for the data sources (cohorts). The joint effect between sleeping difficulty and shift work was only based on the data from NHSII, while the joint effect between sleeping difficulty and sleep apnoea was only based on data from NHS.

We also dichotomised each sleep-related exposure, and then summed these to calculate the number of sleep-related conditions (0 to 4). In NHS, the individual sleep conditions included sleeping difficulty, frequent snoring, sleep duration $\leq 6 \mathrm{~h}$ and sleep apnoea; in NHSII, the individual sleep exposures included in the calculation of total number of sleep conditions included sleeping difficulty, frequent snoring, sleep duration $\leq 6 \mathrm{~h}$ and shift work.

Data were analysed using a commercially available software program (SAS, version 9.3; SAS Institute, Cary, NC, USA), and statistical significance was set at a two-tailed $p<0.05$.

\section{Results}

At baseline, the percentage of women who reported sleeping difficulty (having difficulty falling or staying asleep all or most of the time) was $5.9 \%$ in NHS and $4.8 \%$ in NHSII. Sleeping difficulty was associated with a higher BMI, less physical activity, lower dietary quality score, shorter sleep duration, more frequent snoring, more common use of minor tranquillisers, more frequent excessive daytime sleepiness, higher likelihood of shift work, and higher prevalence of hypertension and depression symptoms, as shown in Table 1. 
Table 1 Baseline characteristics according to the frequency of having difficulty falling asleep or staying asleep

\begin{tabular}{|c|c|c|c|c|c|c|}
\hline \multirow[t]{2}{*}{ Study } & \multicolumn{6}{|c|}{ Frequency of having difficulty falling asleep or staying asleep } \\
\hline & $\begin{array}{l}\text { None of } \\
\text { the time }\end{array}$ & $\begin{array}{l}\text { A little of } \\
\text { the time }\end{array}$ & $\begin{array}{l}\text { Some of } \\
\text { the time }\end{array}$ & $\begin{array}{l}\text { A good bit } \\
\text { of the time }\end{array}$ & $\begin{array}{l}\text { Most of } \\
\text { the time }\end{array}$ & $\begin{array}{l}\text { All of the } \\
\text { time }\end{array}$ \\
\hline \multicolumn{7}{|l|}{ NHS (2000) } \\
\hline$n$ & 20,575 & 18,600 & 12,468 & 4156 & 2073 & 755 \\
\hline$\%$ & 35.1 & 31.7 & 21.3 & 7.1 & 3.5 & 1.3 \\
\hline Age, years & 65.0 & 65.3 & 65.9 & 65.4 & 65.2 & 65.1 \\
\hline BMI, $\mathrm{kg} / \mathrm{m}^{2}$ & 26.3 & 26.3 & 26.6 & 26.6 & 27.1 & 27.6 \\
\hline $\mathrm{BMI} \geq 30 \mathrm{~kg} / \mathrm{m}^{2}, \%$ & 19.7 & 19.3 & 21.0 & 22.5 & 25.4 & 28.5 \\
\hline Exercise, $\mathrm{h} /$ week & 1.9 & 1.8 & 1.7 & 1.5 & 1.3 & 1.2 \\
\hline Alcohol, mg/day & 4.9 & 5.4 & 5.7 & 5.8 & 5.3 & 5.4 \\
\hline AHEI & 50.0 & 49.5 & 49.3 & 49.2 & 48.7 & 48.8 \\
\hline Past smoking, $\%$ & 44.0 & 44.7 & 46.8 & 48.5 & 48.4 & 46.3 \\
\hline Current smoking, $\%$ & 10.0 & 8.5 & 8.7 & 8.8 & 11.2 & 10.0 \\
\hline Premenopausal, \% & 1.5 & 1.4 & 1.4 & 1.5 & 1.3 & 1.5 \\
\hline Multivitamin use, $\%$ & 66.4 & 68.5 & 67.5 & 68.3 & 65.0 & 67.6 \\
\hline Regular aspirin use, $\%$ & 42.6 & 44.5 & 44.2 & 43.3 & 42.3 & 44.9 \\
\hline Self-ranking score of SES & 3.7 & 3.8 & 3.9 & 4.0 & 4.1 & 4.2 \\
\hline Family history of diabetes, $\%$ & 26.0 & 26.1 & 27.0 & 25.5 & 27.2 & 30.8 \\
\hline Sleep duration, h/day & 7.4 & 7.2 & 6.9 & 6.3 & 6.1 & 5.9 \\
\hline Sleep duration $\leq 6 \mathrm{~h} /$ day, $\%$ & 15.5 & 21.4 & 37.4 & 61.9 & 72.1 & 78.4 \\
\hline Frequent snoring, $\%$ & 17.9 & 16.8 & 18.3 & 19.9 & 23.1 & 27.2 \\
\hline Use of minor tranquillisers, $\%$ & 2.5 & 3.7 & 5.5 & 9.2 & 13.6 & 15.6 \\
\hline Prevalence of hypertension, $\%$ & 45.8 & 48.1 & 51.4 & 53.7 & 55.8 & 62.1 \\
\hline Prevalence of depression symptom, $\%$ & 11.1 & 11.9 & 14.8 & 19.9 & 28.5 & 35.8 \\
\hline \multicolumn{7}{|l|}{ NHS II (2001) } \\
\hline$n$ & 28,454 & 22,841 & 13,177 & 5840 & 3277 & 1137 \\
\hline$\%$ & 38.1 & 30.6 & 17.6 & 7.8 & 4.4 & 1.5 \\
\hline Age, years & 45.6 & 46.2 & 46.5 & 46.6 & 46.5 & 46.9 \\
\hline BMI, $\mathrm{kg} / \mathrm{m}^{2}$ & 26.3 & 26.5 & 27.1 & 27.4 & 27.5 & 28.5 \\
\hline $\mathrm{BMI} \geq 30 \mathrm{~kg} / \mathrm{m}^{2}, \%$ & 21.5 & 22.3 & 25.6 & 27.4 & 28.6 & 33.3 \\
\hline Exercise, h/week & 2.5 & 2.5 & 2.3 & 2.2 & 2.0 & 1.8 \\
\hline Alcohol, mg/day & 3.7 & 4.1 & 4.2 & 4.6 & 4.2 & 4.0 \\
\hline AHEI & 48.4 & 48.1 & 47.8 & 47.7 & 47.2 & 46.9 \\
\hline Past smoking, $\%$ & 25.1 & 25.2 & 25.7 & 26.7 & 26.1 & 26.6 \\
\hline Current smoking, $\%$ & 7.6 & 8.0 & 8.8 & 10.4 & 14.2 & 16.0 \\
\hline Premenopausal, \% & 71.3 & 69.6 & 67.9 & 65.2 & 63.1 & 58.0 \\
\hline Multivitamin use, $\%$ & 56.8 & 58.7 & 57.7 & 57.4 & 55.9 & 51.3 \\
\hline Regular aspirin use, $\%$ & 9.7 & 11.3 & 12.1 & 13.5 & 14.9 & 13.6 \\
\hline Self-ranking score of SES & 3.8 & 3.9 & 4.1 & 4.2 & 4.4 & 4.5 \\
\hline Family history of diabetes, $\%$ & 15.0 & 15.7 & 16.3 & 16.9 & 17.0 & 19.4 \\
\hline Sleep duration, h/day & 7.1 & 7.1 & 6.9 & 6.6 & 6.4 & 6.1 \\
\hline Sleep duration $\leq 6 \mathrm{~h} /$ day, $\%$ & 24.0 & 24.0 & 33.4 & 48.0 & 59.7 & 68.6 \\
\hline Frequent snoring, $\%$ & 17.1 & 16.5 & 18.1 & 20.2 & 21.9 & 24.8 \\
\hline Use of minor tranquillisers, $\%$ & 3.1 & 4.9 & 7.3 & 10.2 & 16.0 & 25.9 \\
\hline Shift work, \% & 8.6 & 8.9 & 10.5 & 11.8 & 11.6 & 11.4 \\
\hline Frequent daytime sleepiness, $\%$ & 7.4 & 8.7 & 14.2 & 22.2 & 34.2 & 48.8 \\
\hline Prevalence of hypertension, $\%$ & 16.4 & 18.4 & 21.6 & 24.5 & 27.1 & 33.2 \\
\hline Prevalence of depression symptom, $\%$ & 8.4 & 11.1 & 14.8 & 20.6 & 27.8 & 38.3 \\
\hline
\end{tabular}

Data for the continuous variables are reported as means 
We documented 6,407 cases of type 2 diabetes over a period of up to 10 years of follow-up. Women with sleeping difficulty had an increased risk of type 2 diabetes compared with women without sleeping difficulty (Table 2), which was independent of a variety of risk factors, including menopausal status and hormone use, use of multiple vitamins and aspirin, family history of diabetes, physical activity, dietary quality, alcohol consumption, SES and smoking. In the multivariateadjusted model, comparing women with and without sleeping difficulty, the multivariate-adjusted HRs (95\% CIs) of type 2 diabetes were $1.39(95 \% \mathrm{CI} 1.22,1.59)$ in NHS, $1.46(95 \% \mathrm{CI}$ $1.30,1.64)$ in NHSII and $1.45(95 \%$ CI $1.33,1.58)$ in the pooled analysis (Table 2).

After further adjustment for status of hypertension, depression and BMI individually, the HR between sleeping difficulty and type 2 diabetes fell to 1.34 (95\% CI 1.33, 1.58), 1.34 $(95 \%$ CI $1.22,1.46)$ and 1.34 (95\% CI 1.22, 1.47), respectively. Adjustment for any combination of two or three prespecified metabolic risk factors (obesity, hypertension, depression) further reduced the HR of sleeping difficulty and diabetes compared with adjustment for one factor. When we adjusted for all three factors, the HRs decreased to a value of 1.22 (95\% CI 1.12, 1.34; Fig. 1). The percentage of excessive risk mediated by these three factors together was $46 \%$ (95\% CI $33 \%, 59 \%$ ).

A significant interaction was observed between sleeping difficulty and shift work on risk of type 2 diabetes ( $p$ for multiplicative interaction $=0.08$ and $p$ for additive interaction $=0.04$ ). The multivariate-adjusted HRs of shift work only, sleeping difficulty only and with both conditions were 1.27 (95\% CI 1.14, 1.42), 1.40 (95\% CI 1.23, 1.59) and 2.30 (95\% CI 1.78, 2.96), respectively, as compared with women without sleeping difficulty and never shift work in NHSII (Fig. 2a). The interactions between sleeping difficulty and any other sleep conditions, including frequent snoring, sleep apnoea and short sleep duration were not significant (all $p$ values for interaction $>0.1$; Fig. 2b-d). The multivariate-adjusted HRs of sleep duration $\leq 6 \mathrm{~h}$ only, sleeping difficulty only and with both conditions were 1.13 (95\% CI 1.07, 1.19), 1.32 (95\% CI $1.19,1.55)$ and 1.59 (95\% CI 1.43, 1.76), respectively, as compared with women without sleeping difficulty and sleep duration $>6 \mathrm{~h}$ in the pooled analysis (Fig. 2d).

To further explore whether the effect of sleeping difficulty was independent of other sleep conditions, we simultaneously included all sleep-related factors in the multivariate-adjusted analysis. The multivariate-adjusted HRs between sleeping difficulty and type 2 diabetes were 1.26 (95\% CI 1.10, 1.46) in NHS and 1.28 (95\% CI 1.13, 1.44) in NHS II after simultaneous adjustment for all other sleep-related factors. As compared with women without any of these sleep conditions, women who reported having one (HR: $1.47,95 \%$ CI 1.39 , 1.56), two (HR 2.14, 95\% CI 1.99, 2.31), three (HR 2.84, $95 \%$ CI $2.48,3.26$ ) and all four (HR 4.17, 95\% CI 2.93,
5.91) sleep conditions had gradually increasing HRs of type 2 diabetes ( $p$ trend $<0.0001$ ). In a sensitivity analysis, we also included all potential mediators, confounding factors and sleep conditions in a single model; the simultaneously multivariate-adjusted HR between sleeping difficulty and type 2 diabetes was 1.18 (95\% CI 1.08, 1.29).

Among the relatively healthy population without obesity, hypertension or depression at baseline $(n=82,903,62 \%$ of the sample), the multivariate-adjusted HRs of type 2 diabetes during up to 10 years of follow-up were 1.44 (95\% CI 1.23, 1.74), comparing women with and without sleeping difficulty after adjusting for lifestyle factors, SES and baseline BMI, which decreased to $1.33(95 \%$ CI 1.11, 1.59) after further adjusting for incident hypertension, incident depression and updated BMI. The percentage of excessive risk mediated by these three factors together was $21 \%(95 \%$ CI $9 \%, 41 \%)$ among this relatively healthy population.

\section{Discussion}

In two large prospective cohorts of US women with up to 10 years of follow-up, sleeping difficulty was significantly associated with type 2 diabetes, independent of lifestyle and other sleep-related factors. Our mediation analysis suggested that this positive association was only partially explained by hypertension, BMI and depression. The combination of sleeping difficulty and other sleep conditions including frequent snoring, sleep duration $\leq 6 \mathrm{~h}$, sleep apnoea and shift work was associated with a particularly high risk of diabetes. This paper extends the findings of previous studies in two important ways. First, it is the first study to examine and quantify the mediation effects of chronic conditions on the association between sleeping difficulty and type 2 diabetes. Second, it is the first one to examine the joint effects and test the interactions between sleeping difficulty and other sleeprelated conditions on the risk of type 2 diabetes. The findings provide evidence to clinical physicians and public health researchers for future diabetes prevention among a high risk population with multiple sleep disorders.

Our finding that sleeping difficulty is associated with a higher risk of type 2 diabetes is consistent with previous studies. So far, six studies have addressed the impact of selfreported difficulty falling or staying asleep and the risk of type 2 diabetes [9-12, 24-26]. Half of these studies found a significant positive association between difficulty initiating sleep or maintaining asleep and the risk of type 2 diabetes [10-12, 24, 25], with significant pooled results: 1.57 (95\% CI 1.25, 1.97) for difficulty initiating sleep and $1.84(95 \%$ CI $1.39,2.43)$ for difficulty in maintaining sleep [9]. Our estimation of 1.45 $(95 \%$ CI $1.33,1.58)$ for sleeping difficulty is quite close to the estimation in the above meta-analysis. Our study is, so far, the largest study of sleeping difficulty and type 2 diabetes. 


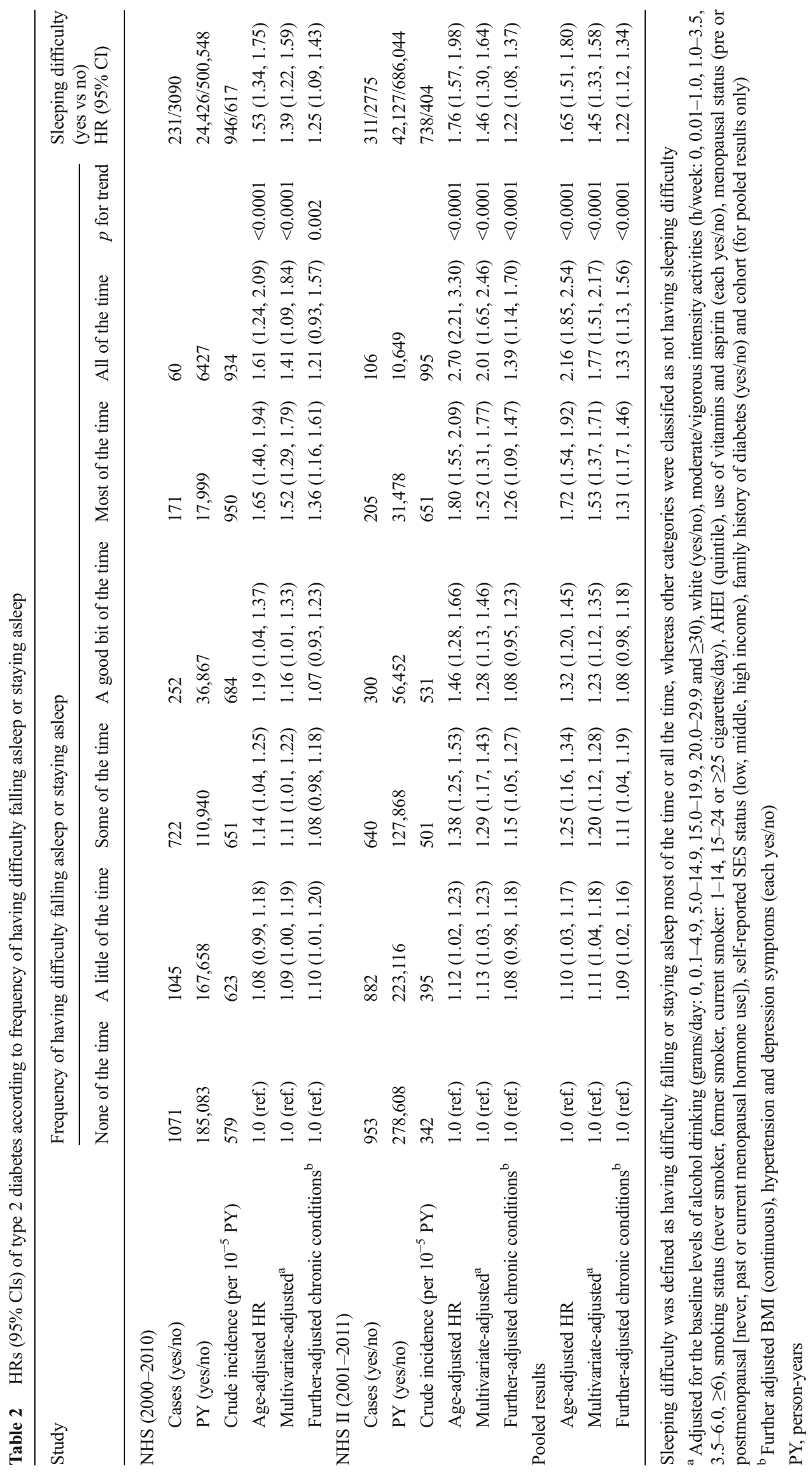




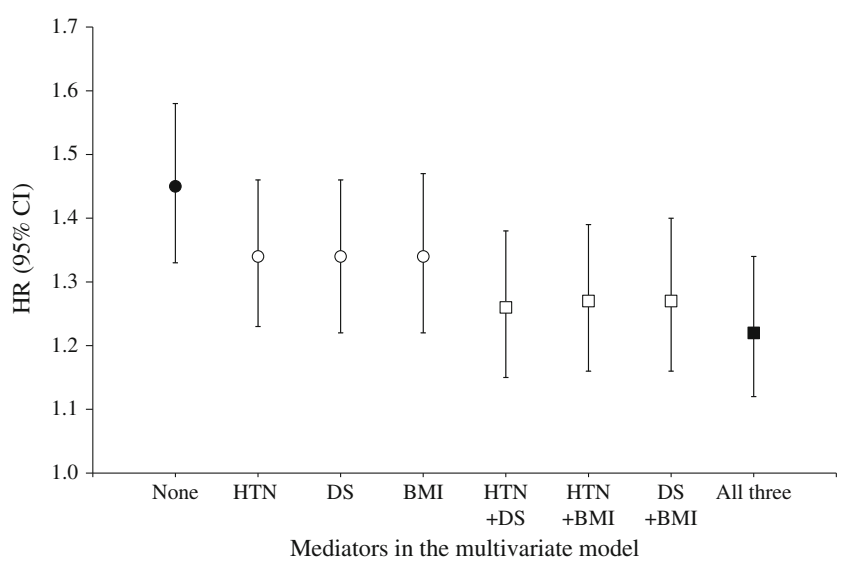

Fig. 1 HRs between sleeping difficulty and type 2 diabetes after further adjustment for different combinations of chronic conditions based on pooled results. The black circle represents adjusting for none of the chronic conditions, white circles represent one chronic condition, white squares represent two chronic conditions, and the black square represents adjusting for all three chronic conditions. All data were adjusted for baseline levels of alcohol drinking, ethnicity, moderate/vigorous intensity activities, smoking status, AHEI, use of vitamins and aspirin, menopausal status, self-reported SES status, family history of diabetes and cohort (for pooled results only). For the specific categories of the variables adjusted for, please refer to the footnotes of Table 2. Sleeping difficulty was defined as having difficulty falling or staying asleep most of the time or all of the time. DS, depression symptoms; HTN, hypertension

Besides the main effects of sleeping difficulty, we also modelled separately with and without different chronic and sleep conditions to explore potential mechanisms.

Several potential mediating pathways were analysed in this study, specifically obesity, hypertension and depression. Sleeping difficulty may influence metabolism and produce obesity by disturbing circadian rhythms and other physiological function [27-29]. Sleep restriction has been shown to be obesogenic, perhaps by altering appetite-regulating hormones [30], which could induce unwanted weight gain [6] and eventually increase the risk of diabetes. Sleep disturbance is also associated with hypertension [7] by increasing blood pressure and sympathetic nervous system activity [31], which may influence insulin sensitivity. As both obesity and hypertension are associated with sleeping difficulty and are risk factors for type 2 diabetes, exploring the potential mediation effect of obesity and hypertension may be helpful for understanding the pathway from sleeping difficulty to type 2 diabetes. Another important factor is depression. Sleep complaints are common in depression [8], and are included in the definition of depression in some cohort studies examining the association of depression and diabetes. Previous studies have shown that depression increases the risk of type 2 diabetes [32]. Our mediation analysis indicated that the sleeping difficulty to diabetes association we identified did not depend exclusively on any specific individual factor or combination of hypertension, BMI or depression; together they explain half of the effect of sleeping difficulty on the risk of type 2 diabetes. The unexplained risk might be caused by other pathways, which warrant future studies.

Other potential pathways that were analysed in the current study were sleep conditions accompanied with sleeping difficulty, specifically short sleep duration, frequent snoring, sleep apnoea and shift work. Sleep continuity is considered to be important for normal metabolic function by modulating or controlling daily patterns in human physiology [27-29]. One of the most common complications of difficulty falling or
Fig. 2 Joint effect of sleeping difficulty and other sleep conditions on the risk of type 2 diabetes. (a) Shift work (NHSII); (b) frequent snoring (NHS and NHSII); (c) sleep apnoea (NHS); and (d) sleep duration $\leq 6 \mathrm{~h} /$ day (NHS and NHSII). Data are presented as $\mathrm{HR} \pm 95 \% \mathrm{CI}$. All data were adjusted for baseline levels of alcohol drinking, ethnicity, moderate/vigorous intensity activities, smoking status, AHEI, use of vitamins and aspirin, menopausal status, selfreported SES status, family history of diabetes and cohort (for pooled results only). For the specific categories of the variables adjusted for, please refer to the footnotes of Table 2
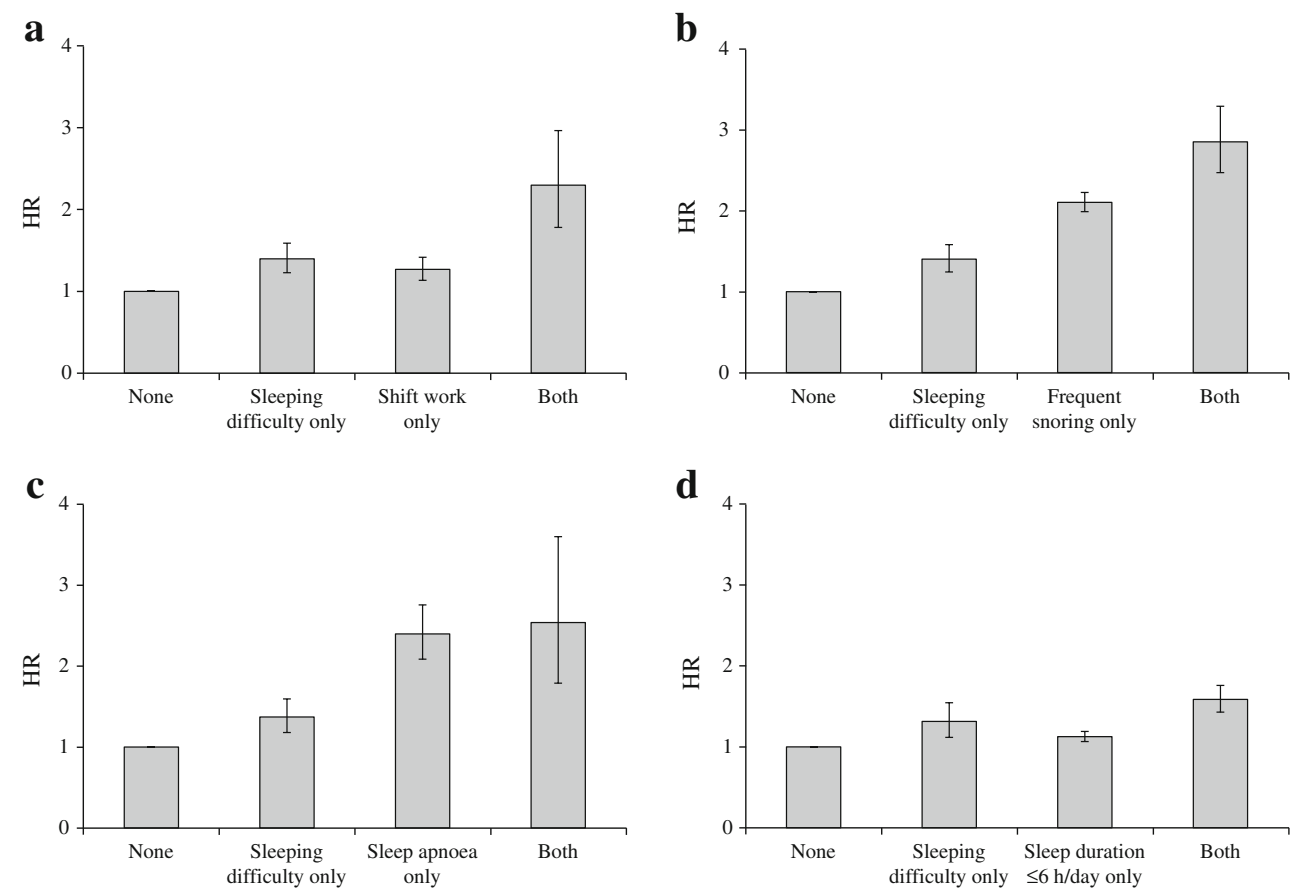
staying asleep is a reduction in night-time sleep $[13,14]$. In our cohorts, more than $60 \%$ of women with sleeping difficulty (defined as all of the time or most of the time) slept less than $7 \mathrm{~h}$ per day, which occurred in only $15-24 \%$ of women without difficulty falling or staying asleep. Experimental sleep restriction reduces glucose tolerance compared with a fully rested condition [4]. A recent meta-analysis of ten prospective studies reported an association between short sleep and incident diabetes of 1.33 (95\% CI 1.20, 1.48) [9]. In our analysis, sleeping difficulty and short sleep duration were independently but also jointly associated with a higher risk of type 2 diabetes. Our previous analysis found a significant positive association between short sleep duration, regular snoring, and rotating shift work and the risk of diabetes [17, 18]. When we added all of the sleep conditions into the model together, the association between sleeping difficulty and risk of type 2 diabetes was attenuated but remained significant, which implied that the pathway between sleeping difficulty and diabetes overlapped partially (but not fully) with other sleep conditions. The present study indicates a particularly high risk of diabetes when sleeping difficulty is combined with other sleep conditions such as short sleep duration, snoring, sleep apnoea and shift work, indicating that these conditions may exacerbate the adverse effects of each other.

Experimental studies have indicated that not only the quantity and quality of sleep, but also the timing of sleep has an effect on glucose regulation [33]. The relative decrease in insulin sensitivity following circadian misalignment and sleep loss was nearly twice as large $(-58 \%)$ as that following the aligned condition (without circadian misalignment) with the same degree of sleep loss only $(-32 \%, p=0.01)$ [34]. Epidemiological studies have also consistently observed significant associations between shift work and the risk of type 2 diabetes. A recent meta-analysis of twelve studies reported a pooled odds ratio of 1.09 (95\% CI 1.05, 1.12) between ever exposure to shift work and the risk of diabetes. In our study, shift work and sleeping difficulty jointly associated with the risk of type 2 diabetes in a manner that was more than additive, which implied that more diabetes cases would occur in women with sleeping difficulty who worked in a shift schedule than in women with only one or the other of these two risk factors. This result is consistent with a recent finding in the NHSII cohort that chronotype (preferred sleep timing) is associated with type 2 diabetes risk, and rotating night shift work significantly modified this effect, with the lowest risks for early chronotype individuals working daytime schedules and latetype chronotype individuals working schedules including night shifts [35].

Strengths of the present study include the large number of incident type 2 diabetes cases, long-term follow-up, consistent findings across two separate cohorts and updated comprehensive information on potential confounders and mediators. Several limitations also warrant consideration. First, assessment of sleeping difficulty was based on self-report, and we did not assess sleep difficulty-related daytime impairment. Objective measurements of sleep onset, maintenance, sleep duration and sleep quality were not available. Sleeping difficulty may occur secondary to obesity [13] or be associated with use of antihypertensive or antidepressant medications. Similarly, it is possible that individuals had sleeping difficulty as a result of undiagnosed depression and that the depression was a residual confounder. We adjusted for these factors, although we cannot totally exclude the potential for residual confounding. A sensitivity analysis among a healthy population without these factors, however, provided a similar estimation of the magnitude of the sleeping difficulty and diabetes association. In addition, our cohorts included mostly white women, potentially limiting the generalisability of the findings. However, the relative homogeneity of the study populations in educational attainment and SES enhances the internal validity.

In conclusion, this study shows that sleeping difficulty is associated with type 2 diabetes, which is partially explained by associations with hypertension, BMI and depression symptoms; the association is particularly strong when combined with other sleep disorders. Our findings highlight the importance of sleep disturbance in the development and prevention of type 2 diabetes.

Acknowledgements We are indebted to the participants in the NHS and NHSII for their continuing outstanding support and colleagues working in this study for their valuable help.

Funding The Study was supported by NIH grants UM1 CA186107 and UM1 CA176726.

Duality of interest The authors declare that there is no duality of interest associated with this manuscript. JWW has received research grant and lecture/other fees from NeuroMetrix (grant only), UCB and Xenoport and SR has received a research grant from Jazz Pharmaceuticals. All have no duality of interest with the current study.

Contribution statement FBH was involved in the conception and design, provision of study materials or patients, the collection and assembly of data, and obtained funding for the study. YL, XG, JWW, EMC, CLJ, ASW, ES, SR and FBH contributed to the analysis and interpretation of the data. YL produced a draft of the article. YL, XG, JWW, EMC, CLJ, ASW, ES, SR and FBH were involved in the critical revision of the article for important intellectual content. YL, XG, JWW, EMC, CLJ, ASW, ES, SR and FBH approved the final article. YL and FBH provided statistical expertise. YL and FBH are responsible for the integrity of the work as a whole.

\section{References}

1. Buysse DJ (2013) Insomnia. JAMA 309:706-716

2. Ohayon MM (2002) Epidemiology of insomnia: what we know and what we still need to learn. Sleep Med Rev 6:97-111

3. Ford ES, Wheaton AG, Cunningham TJ, Giles WH, Chapman DP, Croft JB (2014) Trends in outpatient visits for insomnia, sleep 
apnea, and prescriptions for sleep medications among US adults: findings from the national ambulatory medical care survey 19992010. Sleep 37:1283-1293

4. Spiegel K, Leproult R, van Cauter E (1999) Impact of sleep debt on metabolic and endocrine function. Lancet 354:1435-1439

5. Stamatakis KA, Punjabi NM (2010) Effects of sleep fragmentation on glucose metabolism in normal subjects. Chest 137:95-101

6. Vorona RD, Winn MP, Babineau TW, Eng BP, Feldman HR, Ware JC (2005) Overweight and obese patients in a primary care population report less sleep than patients with a normal body mass index. Arch Intern Med 165:25-30

7. Meng L, Zheng Y, Hui R (2013) The relationship of sleep duration and insomnia to risk of hypertension incidence: a meta-analysis of prospective cohort studies. Hypertens Res 36:985-995

8. Baglioni C, Battagliese G, Feige B, Spiegelhalder K, Nissen C (2011) Insomnia as a predictor of depression: a meta-analytic evaluation of longitudinal epidemiological studies. J Affect Disord 135: $10-19$

9. Cappuccio FP, D'Elia L, Strazzullo P, Miller MA (2010) Quantity and quality of sleep and incidence of type 2 diabetes: a systematic review and meta-analysis. Diabetes Care 33:414-420

10. Kawakami N, Takatsuka N, Shimizu H (2004) Sleep disturbance and onset of type 2 diabetes. Diabetes Care 27:282-283

11. Nilsson PM, Rööst M, Engström G, Hedblad B, Berglund G (2004) Incidence of diabetes in middle-aged men is related to sleep disturbances. Diabetes Care 27:2464-2469

12. Björkelund C, Bondyr-Carlsson D, Lapidus L et al (2005) Sleep disturbances in midlife unrelated to 32-year diabetes incidence: the prospective population study of women in Gothenburg. Diabetes Care 28:2739-2744

13. Jaussent I, Dauvilliers Y, Ancelin ML et al (2011) Insomnia symptoms in older adults: associated factors and gender differences. Am J Geriatr Psychiatry 19:88-97

14. National Sleep Foundation. 2010 sleep in America poll. Available from http://sleepfoundation.org/sites/default/files/nsaw/NSF\% 20Sleep $\% 20$ in $\% 20 \% 20$ America $\% 20$ Poll $\% 20$ - $\% 20$ Summary $\%$ 20of\%20Findings\%20.pdf, accessed 8 Jan 2015

15. Rajaratnam SM, Howard ME, Grunstein RR (2013) Sleep loss and circadian disruption in shift work: health burden and management. Med J Aust 199:S11-S15

16. Jackson CL, Redline S, Kawachi I, Hu FB (2013) Association between sleep duration and diabetes in black and white adults. Diabetes Care 36:3557-3565

17. Al-Delaimy WK, Manson JE, Willett WC, Stampfer MJ, Hu FB (2002) Snoring as a risk factor for type II diabetes mellitus: a prospective study. Am J Epidemiol 155:387-393

18. Pan A, Schernhammer ES, Sun Q, Hu FB (2011) Rotating night shift work and risk of type 2 diabetes: two prospective cohort studies in women. PLoS Med 8: e1001141

19. International Diabetes Federation. IDF diabetes atlas update 2014. Available from www.idf.org/diabetesatlas/update-2014, accessed 8 Jan 2015
20. Hu FB, Leitzmann MF, Stampfer MJ, Colditz GA, Willett WC, Rimm EB (2001) Physical activity and television watching in relation to risk for type 2 diabetes mellitus in men. Arch Intern Med 161:1542-1548

21. Manson JE, Rimm EB, Stampfer MJ et al (1991) Physical activity and incidence of non-insulin-dependent diabetes mellitus in women. Lancet 338:774-778

22. Lin DY, Fleming TR, de Gruttola V (1997) Estimating the proportion of treatment effect explained by a surrogate marker. Stat Med $16: 1515-1527$

23. VanderWeele TJ, Asomaning K, Tchetgen Tchetgen EJ et al (2012) Genetic variants on $15 \mathrm{q} 25.1$, smoking, and lung cancer: an assessment of mediation and interaction. Am J Epidemiol 175:1013-1020

24. Hayashino Y, Fukuhara S, Suzukamo Y, Okamura T, Tanaka T, Ueshima H (2007) Relation between sleep quality and quantity, quality of life, and risk of developing diabetes in healthy workers in Japan: the High-risk and Population Strategy for Occupational Health Promotion (HIPOPOHP) Study. BMC Public Health 7:129

25. Mallon L, Broman JE, Hetta J (2005) High incidence of diabetes in men with sleep complaints or short sleep duration: a 12-year followup study of a middle-aged population. Diabetes Care 28:2762-2767

26. Meisinger C, Heier M, Loewel H, MONICA/KORA Augsburg Cohort Study (2005) Sleep disturbance as a predictor of type 2 diabetes mellitus in men and women from the general population. Diabetologia 48:235-241

27. Morselli LL, Guyon A, Spiegel K (2012) Sleep and metabolic function. Pflugers Arch 463:139-160

28. Depner CM, Stothard ER, Wright KP Jr (2014) Metabolic consequences of sleep and circadian disorders. Curr Diab Rep 14:507

29. Copinschi G, Leproult R, Spiegel K (2014) The important role of sleep in metabolism. Front Horm Res 42:59-72

30. Spiegel K, Tasali E, Penev P, van Cauter E (2004) Brief communication: sleep curtailment in healthy young men is associated with decreased leptin levels, elevated ghrelin levels, and increased hunger and appetite. Ann Intern Med 141:846-850

31. Tochikubo O, Ikeda A, Miyajima E, Ishii M (1996) Effects of insufficient sleep on blood pressure monitored by a new multibiomedical recorder. Hypertension 27:1318-1324

32. Pan A, Lucas M, Sun Q et al (2010) Bidirectional association between depression and type 2 diabetes mellitus in women. Arch Intern Med 170:1884-1891

33. Nedeltcheva AV, Scheer FA (2014) Metabolic effects of sleep disruption, links to obesity and diabetes. Neuroendocrinology 21 : 293-298

34. Buxton OM, Cain SW, O'Connor SP et al (2012) Adverse metabolic consequences in humans of prolonged sleep restriction combined with circadian disruption. Sci Transl Med 4:129ra43

35. Vetter C, Devore EE, Ramin CA, Speizer FE, Willett WC, Schernhammer ES (2015) Mismatch of sleep and work timing and risk of type 2 diabetes. Diabetes Care 38:1707-1713 